\title{
Maximization problem on Trudinger-Moser inequality involving Lebesgue norm
}

Masato Hashizume

\begin{tabular}{|c|l|}
\hline Citation & OCAMI Preprint Series \\
\hline Issue Date & 2018 \\
\hline Type & Preprint \\
\hline Textversion & Author \\
\hline
\end{tabular}

From: Osaka City University Advanced Mathematical Institute http://www.sci.osaka-cu.ac.jp/OCAMI/publication/preprint/preprint.html 


\title{
Maximization problem on Trudinger-Moser inequality involving Lebesgue norm
}

\author{
Masato Hashizume ${ }^{1}$ \\ Graduate School of Science and Engineering, Ehime University \\ 2-5 Bunkyocho, Matsuyamashi, Ehimeken, 790-8577, Japan
}

\begin{abstract}
We study the maximization problem of the Trudinger-Moser inequality. In this study, we consider the effect of the Lebesgue norm on attainability of the best constant. By the Lebesgue norm, there exists a borderline related to existence and non-existence.
\end{abstract}

Keywords: maximization problem, Trudinger-Moser inequality, two dimension, variational problem

\section{Introduction}

Assume that $N \geq 2, \alpha_{N}=N \omega_{N-1}^{\frac{1}{N-1}}$, where $\omega_{N-1}$ is the surface area of $(N-1)$-dimensional unit sphere. The classical Trudinger-Moser inequality asserts that for any bounded domain $\Omega \subset \mathbb{R}^{N}$,

$$
\sup _{\substack{u \in W_{0}^{1, N}(\Omega) \\\|\nabla u\|_{N} \leq 1}} \int_{\Omega} e^{\alpha_{N}|u|^{\frac{N}{N-1}}} d x<+\infty .
$$

Due to [11], $\alpha_{N}$ is the largest possible constant. In [3], they showed that maximizer exists when $\Omega$ is a unit ball. In general bounded domain case, the existence result was shown in 2-dimensional case by [6]. In the $N$-dimensional general bounded domain case, existence of the maximizer was shown by [9]. Recently, [12] was proved the existence of the maximizer by using the blow up analysis different from the technique in [3]. In addition to these, there

\footnotetext{
${ }^{1}$ e-mail:hashizume.masato.ar@ehime-u.ac.jp
} 
are many researches on the Trudinger-Moser inequality. For example, whole space case is due to $[1,14,8,7,4]$, the Trudinger-Moser inequality on $W^{1, N}(\Omega)$ is due to [15], and so on.

In this study, we consider the case when $N=2, \Omega=B$, where $B$ is a unit ball. We investigate existence and non-existence of maximizer for

$$
C(\lambda, p):=\sup _{\substack{u \in H_{0}^{1}(B) \\\|\nabla u\|_{2} \leq 1}} \int_{B}\left(e^{\alpha_{2} u^{2}}-\lambda|u|^{p}\right) d x,
$$

where $\lambda$ is a positive parameter and $p \geq 1$. We can regard this problem as the perturbed Trudinger-Moser inequality

$$
I_{\alpha}^{g}:=\sup _{\substack{u \in H_{0}^{1}(B) \\\|\nabla u\|_{2}^{2} \leq \alpha}} \int_{B}(1+g(u)) e^{u^{2}} d x
$$

and our problem is the case when $\alpha=\alpha_{2}$ and $g(u)=-\lambda \frac{|u|^{p}}{\alpha_{2}^{p / 2} e^{u^{2}}}$. In [12], they consider $I_{\alpha}^{g}$ under the following conditions:

$$
\alpha \leq \alpha_{2}, \quad g \in C^{1}(\mathbb{R}), \quad \inf _{\mathbb{R}} g>-1, \quad g(t)=g(-t), \quad \lim _{|t| \rightarrow \infty} g(t)=0 .
$$

For example, $g \equiv 0$ satisfies the above conditions, and thus the maximization problem on the classical Trudinger-Moser inequality is included this. They studied the blow up analysis for $H_{0}^{1}(B)$-norm of the non-compact sequence on the Trudinger-Moser functional. Their results are useful to study the maximizing problem $I_{\alpha}^{g}$. In our problem, the perturbation $g$ does not satisfy the condition $\inf _{\mathbb{R}} g>-1$. This fact causes the problem that there is the possibility of $I_{\alpha}^{g}=I_{\alpha_{2}}^{g}$ for some $\alpha<\alpha_{2}$, because the function $e^{\alpha_{2}|\cdot|^{2}}-\lambda|\cdot|^{p}$ is not a increasing function.

In [5], they studied the case when $p=2$. They showed existence of maximizer for $\lambda<\alpha_{2}$ and they supposed that the maximizer does not exists for $\lambda \geq \alpha_{2}$. Here, we can see that this term $\alpha_{2}|\cdot|^{2}$ in their conjecture is the second term of $e^{\alpha_{2}|\cdot|^{2}}$, that is, we observe

$$
e^{\alpha_{2}|\cdot|^{2}}=1+\alpha_{2}|\cdot|^{2}+\sum_{k=2}^{\infty} \frac{\alpha_{2}^{k}|\cdot|^{2 k}}{k !},
$$

and the term is appeared. We forecast that there is a relation between this term and the attainability of the maximization problem. Therefore, we 
consider the maximization problem $C(\lambda, p)$, and we study the effect of the $L^{p}$-term. The main theorem is as follows:

Theorem 1.1. (I) If $p>2$, then for any $\lambda$, there exists a maximizer of $C(\lambda, p)$.

(II) If $p \in[1,2]$, then there exists a positive constant $\lambda_{*}=\lambda_{*}(p)$ such that

(i) For $\lambda<\lambda_{*}$, there exists a maximizer of $C(\lambda, p)$.

(ii) For $\lambda>\lambda_{*}$, maximizer of $C(\lambda, p)$ does not exist.

(III) If $p \in[1,2)$, then there exists a maximizer of $C\left(\lambda_{*}, p\right)$, where $\lambda_{*}$ is a positive constant obtained in the part (II).

From these results, we can see that 2 is the borderline on the exponent $p$ essentially. In addition, we can show the conjecture of [5] partially. In order to prove this theorem, we use the blow up analysis. We apply the techniques in [8], [15], and [12]. The techniques of [8], [15] are similar. However, the strategy of [12] are different from those of the others. In [8] and [15], they study the behavior of the Trudinger-Moser functional on the concentrating sequence. On the other hand, in [12], as we referred to before, they investigated the behavior of $H_{0}^{1}(B)$-norm of the concentrating sequence. We will combine these two techniques.

This paper is organized as follows. In Section 2, we prepare some lemmas and propositions. Especially, we investigate the properties of $C(\lambda, p)$ on the parameter $\lambda$. In Section 3, we prove the theorem. To prove this, we prepare two important propositions. In Section 4, we show these two propositions by applying the technique in [8], [15], and [12].

\section{Preliminaries}

First, we fix some notation. The $L^{q}(B)$-norm is written as $\|\cdot\|_{q}$. The constant $C_{0}$ is defined by $C(0, p)$. For simplicity, sometimes we write $v(r)$ as the radially symmetric function $v(x)$ by supposing that $r=|x|$. For a function $v$, we define $v_{+}$and $v_{-}$as $v_{+}:=\max \{v, 0\}$ and $v_{-}:=\min \{v, 0\}$.

We prepare some lemmas and proposition to prove Theorem 1.1. We set

$$
C_{\text {rad }}(\lambda, p)=\sup _{\substack{u \in H_{0, \text { rad }}^{1}(B) \\\|\nabla u\|_{2} \leq 1}} \int_{B}\left(e^{\alpha_{2} u^{2}}-\lambda|u|^{p}\right) d x
$$

where $H_{0, \text { rad }}^{1}(B)$ is the set of radially symmetric functions in $H_{0}^{1}(B)$. By the symmetrization of function in $H_{0}^{1}(B)$, we can see that $C(\lambda, p)=C_{\text {rad }}(\lambda, p)$ 
and existence of maximizer of $C(\lambda, p)$ is equivalent to existence of maximizer of $C_{\text {rad }}(\lambda, p)$.

We take a sequence $\left\{u_{n}\right\}$ satisfying

$$
\begin{gathered}
\left\{u_{n}\right\} \subset H_{0, \text { rad }}^{1}(B), \quad\left\|\nabla u_{n}\right\|_{2} \leq 1, \quad u_{n} \rightarrow 0 \text { weakly in } H_{0}^{1}(B) \\
\lim _{n \rightarrow \infty}\left\|\nabla u_{n}\right\|_{2} \rightarrow 1, \quad \lim _{n \rightarrow \infty}\left\|\nabla u_{n}\right\|_{L^{2}\left(B \backslash B_{\varepsilon}\right)}=0 \quad \text { for any } \varepsilon>0 .
\end{gathered}
$$

We call $\left\{u_{n}\right\}$ satisfying (1), (2) a normalized concentrating sequence. Then we have the following upper bound:

Proposition 2.1 ([3]). For any normalized concentrating sequence $\left\{u_{n}\right\}$, we have

$$
\limsup _{n \rightarrow \infty} \int_{B} e^{\alpha_{2} u_{n}^{2}} d x \leq|B|+e \pi .
$$

Proposition $2.2([5])$. There exists a normalized concentrating sequence $\left\{y_{n}\right\}$ such that

$$
\lim _{n \rightarrow \infty} \int_{B} e^{\alpha_{2} y_{n}^{2}} d x=|B|+e \pi .
$$

More precisely, for sufficiently large $n, y_{n}$ satisfies

$$
\int_{B} e^{\alpha_{2} y_{n}^{2}} d x=|B|+e \pi+\varepsilon_{n}
$$

where $\varepsilon_{n}$ is a positive constant such that $\varepsilon_{n} \rightarrow 0$ as $n \rightarrow \infty$.

The following lemma follows from the definition of $C(\lambda, p)$ and Proposition 2.2 .

Lemma 2.3. (i) $C(\lambda, p)$ is continuous and non-increasing with respect to $\lambda$.

(ii) We have $C(\lambda, p) \geq|B|+$ ex for any $\lambda$ and $p$.

Proposition 2.4. For any $t \in[0,1)$, we have

$$
\sup _{\substack{u \in H_{0}^{1}(B) \\\|\nabla u\|_{2} \leq t}} \int_{B}\left(e^{\alpha_{2} u^{2}}-\lambda|u|^{p}\right) d x<C(\lambda, p) .
$$


Proof. By the part (ii) of Lemma 2.3, we can see that 0 is not maximizer. Set

$$
C_{t}(\lambda, p)=\sup _{\substack{u \in H_{0}^{1}(B) \\\|\nabla u\|_{2} \leq t}} \int_{B}\left(e^{\alpha_{2} u^{2}}-\lambda|u|^{p}\right) d x
$$

and assume that $C_{t}(\lambda, p)=C(\lambda, p)$. We take a maximizing sequence $\left\{u_{n}\right\} \subset$ $H_{0}^{1}(B)$, that is,

$$
\left\|\nabla u_{n}\right\|_{2} \leq t, \quad \lim _{n \rightarrow \infty} \int_{B}\left(e^{\alpha_{2} u_{n}^{2}}-\lambda\left|u_{n}\right|^{p}\right) d x=C(\lambda, p) .
$$

Then we have $u_{n} \rightarrow u_{\infty}$ weakly in $H_{0}^{1}(B)$ and $\left\|\nabla u_{\infty}\right\|_{2}=\tilde{t} \leq t$. Moreover, by the compactness of the Trudinger-Moser functional and the Sobolev embedding, it follows that

$$
\int_{B}\left(e^{\alpha_{2} u_{\infty}^{2}}-\lambda\left|u_{\infty}\right|^{p}\right) d x=\lim _{n \rightarrow \infty} \int_{B}\left(e^{\alpha_{2} u_{n}^{2}}-\lambda\left|u_{n}\right|^{p}\right) d x=C(\lambda, p) .
$$

In addition, we may assume that $u_{\infty} \geq 0$. Since $u_{\infty}$ is also the maximizer of

$$
\sup _{\substack{u \in H_{0}^{1}(B) \\\|\nabla u\|_{2}=\tilde{t}}} \int_{B}\left(e^{\alpha_{2} u^{2}}-\lambda|u|^{p}\right) d x
$$

there exists the Lagrange multiplier $L$ such that

$$
L \int_{B} \nabla u_{\infty} \nabla \phi d x-\int_{B}\left(\alpha_{2} u_{\infty} e^{\alpha_{2} u_{\infty}^{2}}-\frac{p}{2} \lambda u_{\infty}^{p-1}\right) \phi d x=0
$$

for any $\phi \in H_{0}^{1}(B)$. On the other hand, for $s \in[0,1 / \tilde{t}]$ we set

$$
f(s):=\int_{B}\left[e^{\alpha_{2}\left(s u_{\infty}\right)^{2}}-\lambda\left(s\left|u_{\infty}\right|\right)^{p}\right] d x .
$$

Then since $\left.f^{\prime}(s)\right|_{s=1}=0$ we have

$$
\int_{B}\left(2 \alpha_{2} u_{\infty}^{2} e^{\alpha_{2} u_{\infty}^{2}}-p \lambda u_{\infty}^{p}\right) d x=0,
$$

and hence $L=0$. From this, it follows that

$$
e^{\alpha_{2} u_{\infty}^{2}}-\frac{p}{2 \alpha_{2}} \lambda u_{\infty}^{p-2}=0 .
$$

for any $x \in B$. However, for any $\lambda$ and $p$, this equality does not hold for $x$ near $\partial B$ since $\left.u_{\infty}\right|_{\partial B}=0$. 
Lemma 2.5. (i) If $C(\lambda, p)>|B|+$ en, then maximizer of $C(\lambda, p)$ exists.

(ii) If there exists $\lambda_{*}$ such that $C\left(\lambda_{*}, p\right)=|B|+e \pi$, then for $\lambda>\lambda_{*}$ maximizer does not exist.

Proof. We prove (i). Assume that $\left\{u_{n}\right\}$ is a maximizing sequence of $C(\lambda, p)$, namely, $\left\{u_{n}\right\}$ satisfies

$$
\left\{u_{n}\right\} \subset H_{0, \text { rad }}^{1}(B), \quad\left\|\nabla u_{n}\right\|_{2} \leq 1, \quad \lim _{n \rightarrow \infty} \int_{B}\left(e^{\alpha_{2} u_{n}^{2}}-\lambda\left|u_{n}\right|^{p}\right) d x=C(\lambda, p) .
$$

Since $\left\{u_{n}\right\}$ is bounded sequence, there exists $u_{\infty}$ such that up to a subsequence $u_{n} \rightarrow u_{\infty}$ weakly in $H_{0}^{1}(B)$, and $\left\|\nabla u_{\infty}\right\|_{2} \leq 1$. By the assumption and Proposition 2.1, we can see that $\left\{u_{n}\right\}$ is not normalized concentrating sequence. Therefore by the theorem in [10] and the Sobolev embedding, we have

$$
\lim _{n \rightarrow \infty} \int_{B}\left(e^{\alpha_{2} u_{n}^{2}}-\lambda\left|u_{n}\right|^{p}\right) d x=\int_{B}\left(e^{\alpha_{2} u_{\infty}^{2}}-\lambda\left|u_{\infty}\right|^{p}\right) d x .
$$

Consequently $u_{\infty}$ is the maximizer.

We prove (ii). Assume that $\lambda>\lambda_{*}$ and $u_{\lambda} \in H_{0, \text { rad }}^{1}(B)$ is a maximizer of $C(\lambda, p)$. Then we have

$$
\begin{aligned}
|B|+e \pi & \leq C(\lambda, p)=\int_{B}\left(e^{\alpha_{2} u_{\lambda}^{2}}-\lambda\left|u_{\lambda}\right|^{p}\right) d x \\
& <\int_{B}\left(e^{\alpha_{2} u_{\lambda}^{2}}-\lambda_{*}\left|u_{\lambda}\right|^{p}\right) d x \leq C\left(\lambda_{*}, p\right)=|B|+e \pi .
\end{aligned}
$$

This is a contradiction.

Proposition 2.6 (Proposition B.1 in [2]). We define the function with positive parameter $t$, which is introduced in [11] originally.

$$
m_{t}(r)=t^{\frac{1}{2}} m_{1}\left(r^{\frac{1}{t}}\right)=\omega_{1}^{-\frac{1}{2}}(\log 2)^{\frac{1}{2}} t^{\frac{1}{2}} \min \left\{\frac{\log \frac{1}{r}}{t(\log 2)}, 1\right\},
$$

where

$$
m_{1}(r):=\omega_{1}^{-\frac{1}{2}}(\log 2)^{\frac{1}{2}} \min \left\{\frac{\log \frac{1}{r}}{\log 2}, 1\right\} .
$$

Assume that $u_{n} \in H_{0, \text { rad }}^{1}(B)$ satisfying $\left\|\nabla u_{n}\right\| \leq 1, u_{n} \rightarrow 0$ weakly in $H_{0}^{1}(B)$. In addition, $u_{n}$ satisfies

$$
\liminf _{n \rightarrow \infty} \int_{B} e^{\alpha_{2} u_{n}^{2}} d x>|B|
$$


Then, there exists a sequence $\left\{t_{n}\right\} \subset(0,1)$ such that

$$
u_{n}-m_{t_{n}} \rightarrow 0 \text { strongly in } H_{0}^{1}(B) .
$$

Remark 2.1. In [2], they wrote that this proposition holds for all dimension. However, the author can confirm the validity of this proposition only when $N=2$.

\section{Proof of Theorem 1.1}

In this section, we prove Theorem 1.1. We prepare the following two propositions. We will prove these in the next section.

Proposition 3.1. We assume that $p \in[1,2]$, and that there exists a maximizer of $C(\lambda, p)$ for sufficiently large $\lambda$. We write $u_{\lambda}$ as the maximizer for $\lambda$. Then, there exist positive constants $C_{1}=C_{1}(p), C_{2}=C_{2}(p)$ such that for sufficient large $\lambda$ we have

$$
\left\|\nabla u_{\lambda}\right\|_{2}^{2} \leq 1-\lambda \frac{C_{1}}{\left\|u_{\lambda}\right\|_{\infty}^{p+2}}+\frac{C_{2}}{\left\|u_{\lambda}\right\|_{\infty}^{4}}+o\left(\left\|u_{\lambda}\right\|_{\infty}^{-4}\right) .
$$

Proposition 3.2. We fix $\lambda$ and $p$. Assume that $u_{k}$ is a maximizer of

$$
C_{k}(\lambda, p):=\sup _{\substack{u \in H_{0}^{1}(B) \\\|\nabla u\|_{2} \leq 1}} \int_{B}\left(e^{\alpha_{k} u^{2}}-\lambda|u|^{p}\right) d x,
$$

where $\alpha_{k}$ is a sequence of real numbers such that $\alpha_{k} \nearrow \alpha_{2}$ as $k \rightarrow \infty$. If $\sup _{x \in B} u_{k}(x)=u(0) \rightarrow \infty$ as $k \rightarrow \infty$, then there exists a positive constant $C=C(p)$ such that

$$
\left\|\nabla u_{k}\right\|_{2}^{2} \geq \frac{\alpha_{2}}{\alpha_{k}}\left(1+\frac{1}{\alpha_{2}^{2}} \frac{1}{\left\|u_{k}\right\|_{\infty}^{4}}-\lambda \frac{C}{\left\|u_{k}\right\|_{\infty}^{p+2}}\right)+o\left(\left\|u_{k}\right\|_{\infty}^{-4}\right) .
$$

First, we prove the part (I). Since it follows that

$$
\lim _{\varepsilon \rightarrow 0} \sup _{\substack{u \in H_{0}^{1}(B) \\\|\nabla u\|_{2} \leq 1}} \int_{B}\left(e^{\left(\alpha_{2}-\varepsilon\right) u^{2}}-\lambda|u|^{p}\right) d x=C(\lambda, p),
$$

$u_{k}$ defined in Proposition 3.2 is a maximizing sequence of $C(\lambda, p)$. In addition, since $\left\|\nabla u_{k}\right\|_{2} \leq 1$, there exists $u_{\infty} \in H_{0}^{1}(B)$ such that up to a subsequence 
$u_{n} \rightarrow u_{\infty}$ weakly in $H_{0}^{1}(B)$, and $\left\|\nabla u_{\infty}\right\|_{2} \leq 1$. For $p>2$, if $\sup _{x \in B} u_{k}(x) \rightarrow$ $\infty$ as $k \rightarrow \infty$, then $\left\|\nabla u_{k}\right\|_{2}^{2}>1$ for sufficient large $k$ by Proposition 3.2, which contradicts that $\left\|\nabla u_{k}\right\|_{2} \leq 1$. Thus $\sup _{x \in B} u_{k}(x)$ is bounded for $k$ uniformly. By the dominated convergence theorem, we have

$\int_{B}\left(e^{\alpha_{2} u_{\infty}^{2}}-\lambda\left|u_{\infty}\right|^{p}\right) d x=\lim _{k \rightarrow \infty} \int_{B}\left(e^{\alpha_{k} u_{k}^{2}}-\lambda\left|u_{k}\right|^{p}\right) d x=\lim _{k \rightarrow \infty} C_{k}(\lambda, p)=C(\lambda, p)$.

Consequently $u_{\infty}$ is the maximizer of $C(\lambda, p)$.

Next, we prove the part (II). In order to show existence of $\lambda_{*}$ we show nonexistence of maximizer for large $\lambda$. For $p \in[1,2]$, we assume that $u_{\lambda}$ is a maximizer for sufficiently large $\lambda$. By Proposition 2.4, we have $\left\|\nabla u_{\lambda}\right\|_{2}=1$. However, by Proposition 3.1 we have $\left\|\nabla u_{\lambda}\right\|_{2}<1$ for sufficiently large $\lambda$, which is a contradiction. Therefore maximizer does not exists for sufficiently large $\lambda$. By part (i) of Lemma 2.5 we can find that $C(\lambda, p)=|B|+e \pi$ for sufficiently large $\lambda$. We define $\lambda_{*} \in[0, \infty)$ as

$$
\lambda_{*}:=\inf \{\lambda>0|C(\lambda, p)=| B \mid+e \pi\} .
$$

By Lemma 2.3, we can see that $C(\lambda, p)=|B|+e \pi$ for $\lambda \geq \lambda_{*}$ and $C(\lambda, p)>$ $|B|+e \pi$ for $\lambda \in\left(0, \lambda_{*}\right)$. Consequently, this $\lambda_{*}$ is the borderline of the result of part (II) by Lemma 2.5. To finish the proof of the part (II), we have to confirm that $\lambda_{*}>0$. By Proposition 2.2, we have $C_{0}>|B|+e \pi$. Thus we have $C(\lambda, p)>|B|+e \pi$ for sufficiently small $\lambda$ and hence $\lambda_{*}>0$.

Finally, we prove the part (III). We set a sequence $\lambda_{n}$ such that $\lambda_{n} \nearrow \lambda_{*}$ as $n \rightarrow \infty$. By the part (II), maximizer of $C\left(\lambda_{n}, p\right)$ exists and we write this as $u_{n}$. By the part of (i) of Lemma 2.3, we can see that $u_{n}$ is a maximizing sequence of $C\left(\lambda_{*}, p\right)$. If $\sup _{x \in B} u_{n}(x) \rightarrow \infty$ as $\lambda \rightarrow \infty$, we can show that $\left\|\nabla u_{n}\right\|_{2}<1$ for sufficiently large $n$ by preparing similar proposition to Proposition 3.1. This contradicts that $\left\|\nabla u_{n}\right\|_{2}=1$. Consequently $\sup _{x \in B} u_{n}(x)$ is bounded uniformly for sufficiently large $n$, and by the dominated convergence theorem we can show existence of maximizer.

\section{Proof of Proposition 3.1 and 3.2}

Since the proof of Proposition 3.2 is close to the proof of Proposition 3.1 we only prove Proposition 3.1. Fix $p \in[1,2]$ and set a sequence $\lambda_{k}$ such that $\lambda_{k} \rightarrow \infty$ as $k \rightarrow \infty$ (a suitable subsequence is also written by $\lambda_{k}$ ). Assume 
that $u_{k}:=u_{\lambda_{k}} \in H_{0}^{1}(B)$ is the maximizer of $C\left(\lambda_{k}, p\right)$. From Proposition 2.4, we have

$$
\left\|\nabla u_{k}\right\|_{2}=1, \quad u_{k} \in H_{0, r a d}^{1}(B), \quad u_{k} \geq 0, \quad \frac{\partial u_{k}}{\partial r} \text { is a decreasing function. }
$$

By the part (ii) of Lemma 2.3 we have

$$
|B|+e \pi \leq \lim _{k \rightarrow \infty} \int_{B}\left(e^{\alpha_{2} u_{k}^{2}}-\lambda_{k}\left|u_{k}\right|^{p}\right) d x \leq C(0, p)-\lim _{k \rightarrow \infty} \lambda_{k} \int_{B}\left|u_{k}\right|^{p} d x .
$$

Thus up to a subsequence

$$
\int_{B}\left|u_{k}\right|^{p} d x=O\left(\lambda_{k}^{-1}\right), \quad u_{k}(x) \rightarrow 0 \quad \text { for } x \in B \backslash\{0\} .
$$

In addition, since

$$
|B|+e \pi \leq \lim _{k \rightarrow \infty} \int_{B}\left(e^{\alpha_{2} u_{k}^{2}}-\lambda_{k}\left|u_{k}\right|^{p}\right) d x \leq \lim _{k \rightarrow \infty} \int_{B} e^{\alpha_{2} u_{k}^{2}} d x
$$

we have

$$
\lim _{k \rightarrow \infty} \sup _{x \in B} u_{k}=\lim _{k \rightarrow \infty} u_{k}(0)=\infty .
$$

By the Lagrange multiplier theorem, $u_{k}$ is a solution of

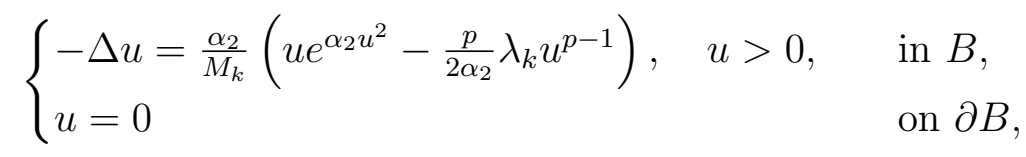

where

$$
M_{k}:=\alpha_{2} \int_{B}\left(u_{k}^{2} e^{\alpha_{2} u_{k}^{2}}-\frac{p}{2 \alpha_{2}} \lambda_{k} u_{k}^{p}\right) d x .
$$

By setting $v_{k}:=\alpha_{2}^{1 / 2} u_{k}, v_{k}$ satisfies

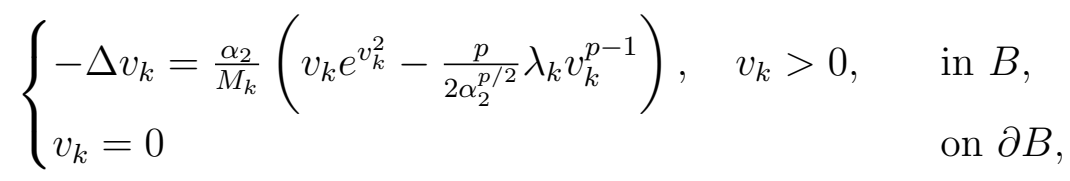

and

$$
\left\|\nabla v_{k}\right\|_{2}^{2}=\alpha_{2}, \quad M_{k}=\int_{B}\left(v_{k}^{2} e^{v_{k}^{2}}-\frac{p}{2 \alpha_{2}^{p / 2}} \lambda_{k} v_{k}^{p}\right) d x .
$$

We note that $\lim _{k \rightarrow \infty} v_{k}=0$ in $B \backslash\{0\}$ and $\lim _{k \rightarrow \infty} v_{k}(0)=\infty$. For simplicity, we set

$$
c_{k}:=v_{k}(0)=\sup _{x \in B} v_{k}(x)
$$


Lemma 4.1. We have

$$
\lim _{k \rightarrow \infty} \lambda_{k} \int_{B} v_{k}^{p} d x=0 .
$$

Proof. We show that $\lim _{k \rightarrow \infty} \lambda_{k} \int_{B} u_{k}^{p} d x=0$. By the part (ii) of Lemma 2.3 and (3), we can find that $u_{k}$ is a normalized concentrating sequence. Thus by Proposition 2.1, it follows that

$$
\lim _{k \rightarrow \infty} \int_{B} e^{\alpha_{2} u_{k}^{2}} d x=|B|+e \pi .
$$

This observation and (3) yield the equality of the lemma.

Lemma 4.2. For sufficient large $k$, we have

$$
M_{k}>\alpha_{2} e \pi+o(1) .
$$

Proof. By the part (ii) of Lemma 2.3 and Lemma 4.1, we have

$$
\begin{aligned}
|B|+e \pi & \leq \int_{B}\left(e^{\alpha_{2} u_{k}^{2}}-\lambda_{k} u_{k}^{p}\right) d x \\
& \leq \int_{\left[u_{k} \leq 1\right]} e^{\alpha_{2} u_{k}^{2}} d x+\int_{\left[u_{k}>1\right]} e^{\alpha_{2} u_{k}^{2}} d x-\lambda_{k} \int_{B} u_{k}^{p} d x \\
& \leq|B|+o(1)+\int_{\left[u_{k}>1\right]} u_{k}^{2} e^{\alpha_{2} u_{k}^{2}} d x-\lambda_{k} \int_{B} u_{k}^{p} d x \\
& \leq|B|+\frac{M_{k}}{\alpha_{2}}+o(1) .
\end{aligned}
$$

Lemma 4.3. For sufficiently large $k$, we have

$$
M_{k} \leq c_{k}^{2}(|B|+e \pi+o(1)) .
$$

Proof. By Lemma 4.1, we have

$$
\begin{aligned}
M_{k} & =\int_{B}\left(v_{k}^{2} e^{v_{k}^{2}}-\frac{p}{2 \alpha_{2}^{p / 2}} \lambda_{k} v_{k}^{p}\right) d x \\
& \leq c_{k}^{2} \int_{B} e^{v_{k}^{2}} d x+o(1) \\
& =c_{k}^{2}(|B|+e \pi+o(1)) .
\end{aligned}
$$


We set

$$
r_{k}:=\frac{\sqrt{M_{k}}}{\sqrt{\pi} c_{k}} e^{-\frac{c_{k}^{2}}{2}}
$$

and

$$
\left\{\begin{array}{l}
\phi_{k}(y):=c_{k}\left(v_{k}\left(r_{k} y\right)-c_{k}\right), \\
\psi_{k}(y):=c_{k}^{-1} v_{k}\left(r_{k} y\right)
\end{array}\right.
$$

Then, we have

$$
\begin{aligned}
& -\Delta \phi_{k}=4\left[\psi_{k} e^{\phi_{k}\left(1+\psi_{k}\right)}-\frac{p}{2 \alpha_{2}^{p / 2}} c_{k}^{p-2} e^{-c_{k}^{2}} \lambda \psi_{k}^{p-1}\right] \quad \text { in } B_{1 / r_{k}} \\
& -\Delta \psi_{k}=\frac{4}{c_{k}^{2}}\left[\psi_{k} e^{c_{k}^{2}\left(\psi_{k}-1\right)}-\frac{p}{2 \alpha_{2}^{p / 2}} c_{k}^{p-2} e^{-c_{k}^{2}} \lambda_{k} \psi_{k}\right] \quad \text { in } B_{1 / r_{k}}
\end{aligned}
$$

Multiplying (7) by $c_{k}^{2} \psi_{k}$, integrating on $B_{1 / r_{k}}$, and we have

$$
\alpha_{2}=4\left[\int_{B_{1 / r_{k}}} \psi_{k}^{2} e^{c_{k}^{2}\left(\psi_{k}^{2}-1\right)} d x-\frac{p}{2 \alpha_{2}^{p / 2}} c_{k}^{p-2} e^{-c_{k}^{2}} \lambda_{k} \int_{B_{1 / r_{k}}} \psi_{k}^{2} d x\right],
$$

and thus

$$
\frac{2 p}{\alpha_{2}^{p / 2}} c_{k}^{p-2} e^{-c_{k}^{2}} \lambda_{k}=\frac{4 \int_{B_{1 / r_{k}}} \psi_{k}^{2} e^{c_{k}^{2}\left(\psi_{k}^{2}-1\right)} d x-\alpha_{2}}{\int_{B_{1 / r_{k}}} \psi_{k}^{2} d x} \leq 4-\frac{\alpha_{2}}{\int_{B_{1 / r_{k}}} \psi_{k}^{2} d x} .
$$

Thus we can use the elliptic regularity theory. We have

$$
\psi_{k} \rightarrow 1 \quad \text { in } C_{l o c}^{2}\left(\mathbb{R}^{2}\right)
$$

On the other hand, in (8), by Lemma 4.1 we have

$4 \int_{B_{1 / r_{k}}} \psi_{k}^{2} e^{c_{k}^{2}\left(\psi_{k}^{2}-1\right)} d x=\frac{\alpha_{2}}{M_{k}} \int_{B} v_{k}^{2} e^{v_{k}^{2}} d x=\alpha_{2}+\frac{\alpha_{2}}{M_{k}} \frac{p}{2 \alpha_{2}^{p / 2}} \lambda_{k} \int_{B} v_{k}^{p}=\alpha_{2}+o(1)$, and by (9)

$$
\lim _{k \rightarrow \infty} \int_{B_{1 / r_{k}}} \psi_{k}^{2} d x \geq \lim _{R \rightarrow \infty} \lim _{k \rightarrow \infty} \int_{B_{R}} \psi_{k}^{2} d x=\lim _{R \rightarrow \infty}\left|B_{R}\right|=\infty .
$$


Hence

$$
\frac{p}{2 \alpha_{2}^{p / 2}} c_{k}^{p-2} e^{-c_{k}^{2}} \lambda_{k}=o(1) .
$$

Concerning the equation (6), by the elliptic regularity theory,

$$
\begin{gathered}
\phi_{k} \rightarrow \phi_{\infty}=-\log \left(1+|x|^{2}\right) \quad \text { in } C_{l o c}^{2}\left(\mathbb{R}^{2}\right), \\
-\Delta \phi_{\infty}=4 e^{2 \phi_{\infty}} \quad \text { in } \mathbb{R}^{2} .
\end{gathered}
$$

For a constant $\rho>1$ we set

$$
v_{k, \rho}:=\min \left\{\frac{c_{k}}{\rho}, v_{k}\right\}
$$

Lemma 4.4.

$$
\lim _{k \rightarrow \infty} \int_{B}\left|\nabla v_{k, \rho}\right|^{2} d x=\frac{\alpha_{2}}{\rho}
$$

The strategy is same as the proof of Lemma 3.3 in [8] and the proof of Lemma 3.6 in [15].

Lemma 4.5. We have

$$
\liminf _{k \rightarrow \infty} \frac{M_{k}}{c_{k}^{2}} \geq e \pi .
$$

Proof. By Lemma 4.4 we have

$$
\begin{aligned}
\int_{B} e^{v_{k}^{2}} d x & =\int_{\left[v_{k}<c_{k} / \rho\right]} e^{v_{k}^{2}} d x+\int_{\left[v_{k} \geq c_{k} / \rho\right]} e^{v_{k}^{2}} d x \\
& \leq \int_{B} e^{v_{k, \rho}^{2}}+\frac{\rho^{2}}{c_{k}^{2}} \int_{B} v_{k}^{2} e^{v_{k}^{2}} d x \\
& =|B|+\frac{\rho^{2}}{c_{\lambda}^{2}} \frac{M_{k}}{\alpha_{2}}\left[\frac{\alpha_{2}}{M_{k}} \int_{B}\left(v_{k}^{2} e^{v_{k}^{2}} d x-\frac{p}{2 \alpha_{2}^{p / 2}} \lambda_{k} v_{k}^{p}\right) d x+\frac{\alpha_{2}}{M_{k}} \frac{p}{2 \alpha_{2}^{p / 2}} \lambda_{k} \int_{B} v_{k}^{p} d x\right]+o(1) \\
& =|B|+\frac{\rho^{2}}{c_{\lambda}^{2}} M_{k}+o(1) .
\end{aligned}
$$

On the left hand side, we have

$$
\int_{B} e^{v_{k}^{2}} d x=\int_{B} e^{\alpha_{2} u_{k}^{2}} d x=|B|+e \pi+o(1) .
$$

Hence we obtain the inequality of the lemma. 
Combining Lemma 4.3 and 4.5, for sufficiently large $k$

$$
e \pi+o(1) \leq \frac{M_{k}}{c_{k}^{2}} \leq|B|+e \pi+o(1)
$$

Lemma 4.6. For any $\phi \in C^{\infty}(B)$ we have

$$
\lim _{k \rightarrow \infty} \frac{1}{M_{k}} \int_{B} c_{k} v_{k} e^{v_{k}^{2}} \phi d x=\phi(0) .
$$

We can prove in the same way as the proof of Lemma 3.6 in [8] and the proof of Lemma 3.9 in [15].

Proposition 4.7. There exists positive constants $C_{1}, C_{2}$ such that for sufficiently large $k$ we have

$$
\frac{C_{1}}{c_{k}^{p}} \leq \int_{B} v_{k}^{p} d x \leq \frac{C_{2}}{c_{k}^{p}}
$$

Proof. We consider the equation:

$$
\begin{cases}-\Delta\left(c_{k} v_{k}\right)=\frac{\alpha_{2}}{M_{k}}\left[\left(c_{k} v_{k}\right) e^{v_{k}^{2}}-\frac{p}{2 \alpha_{2}^{p / 2}} \lambda_{k} c_{k} v_{k}^{p-1}\right] & \text { in } B, \\ v_{k}=0 & \text { on } \partial B .\end{cases}
$$

It follows that

$$
\begin{aligned}
\int_{B}\left(\Delta c_{k} v_{k}\right)_{+} d x & =-\frac{\alpha_{2}}{M_{k}} \int_{\left[\Delta c_{k} v_{k}>0\right]}\left(c_{k} v_{k} e^{v_{k}^{2}}-\frac{p}{2 \alpha_{2}^{p / 2}} \lambda_{k} c_{k} v_{k}^{p}\right) d x \\
& \leq \frac{\alpha_{2}}{M_{k}} \int_{B} \frac{p}{2 \alpha_{2}^{p / 2}} \lambda_{k} c_{k} v_{k}^{p-1} d x \\
& =\alpha_{2}+o(1)-\frac{\alpha_{2}}{M_{\lambda}}\left(\int_{B} c_{k} v_{k} e^{v_{k}^{2}} d x-\int_{B} \frac{p}{2 \alpha_{2}^{p / 2}} \lambda_{k} c_{k} v_{k}^{p-1} d x\right) \\
& =\alpha_{2}+o(1)+\int_{B} \Delta\left(c_{k} v_{k}\right) d x \\
& =\alpha_{2}+o(1)+c_{k} \int_{\partial B} \frac{\partial v_{k}}{\partial \nu} d \sigma \\
& \leq \alpha_{2}+o(1),
\end{aligned}
$$


and

$$
\begin{aligned}
-\int_{B}\left(\Delta c_{k} v_{k}\right)_{-} d x & =\frac{\alpha_{2}}{M_{\lambda}} \int_{\left[\Delta c_{k} v_{k} \leq 0\right]}\left(c_{k} v_{k} e^{v_{k}^{2}}-\frac{p}{2 \alpha_{2}^{p / 2}} \lambda_{k} c_{k} v_{k}^{p-1}\right) d x \\
& \leq \frac{\alpha_{2}}{M_{k}} \int_{B} c_{k} v_{k} e^{v_{k}^{2}} d x \\
& =\alpha_{2}+o(1) .
\end{aligned}
$$

Thus we have $\int_{B}\left|\Delta c_{\lambda} v_{k}\right| d x<2 \alpha_{2}+o(1)$ and hence there exists $w \in W^{2,1}(B)$ such that

$$
c_{k} v_{k} \rightarrow w \quad \text { weakly in } \quad W^{2,1}(B) .
$$

From this,

$$
c_{k} v_{k} \rightarrow w \quad \text { weakly in } \quad W^{1, q}(B)
$$

for any $q \in[1,2)$, and we have

$$
\int_{B}\left(c_{k} v_{k}\right)^{p} d x \rightarrow \int_{B} w^{p} d x
$$

Moreover, the compact embedding yields that

$$
c_{k} v_{k} \rightarrow w \quad \text { strongly in } C^{0, \alpha}\left(B_{1} \backslash B_{\varepsilon}\right)
$$

for any $\varepsilon>0$. To end the proof, we show that $w \neq 0$.

By Proposition 2.6 there exists a sequence $\left\{t_{k}\right\}$ such that

$$
\int_{B}\left|\nabla\left(v_{k}-\alpha_{2}^{1 / 2} m_{t_{k}}\right)\right|^{2} d x \rightarrow 0
$$

where $m_{t_{k}}$ is defined as in Proposition 2.6. In addition, since $H_{0}^{1}$-norm has the scaling invariance, it follows that

$$
\int_{B}\left|\nabla\left(t_{k}^{-\frac{1}{2}} v_{k}\left(|x|^{t_{k}}\right)-\alpha_{2}^{1 / 2} m_{1}(|x|)\right)\right|^{2} d x \rightarrow 0 .
$$

Thus we have

$$
t_{k}^{-\frac{1}{2}} v_{k}\left(r^{t_{k}}\right)-\alpha_{2}^{1 / 2} \omega_{1}^{-\frac{1}{2}}(\log 2)^{\frac{1}{2}} \rightarrow 0
$$

for each $r \in(0,1 / 2)$. Since $v_{k}$ is a radially decreasing function, we can find that $t_{k}=O\left(c_{k}^{2}\right)$. We go back to Proposition 2.6 and it follows that

$$
\int_{B}\left|\nabla\left(\alpha_{2}^{1 / 2} \tilde{m}_{t_{k}}(|x|)-t_{k}^{\frac{1}{2}} v_{k}\left(r^{\frac{1}{t_{k}}}\right)\right)\right|^{2} d x \rightarrow 0,
$$


where

$$
\tilde{m}_{t_{k}}(r)=t_{k}^{\frac{1}{2}} m_{t_{k}}\left(r^{\frac{1}{t_{k}}}\right)=t_{k} m_{1}\left(r^{\frac{1}{t_{k}^{2}}}\right)
$$

From this, we have

$$
t_{k}^{\frac{1}{2}} v_{k}\left(r^{\frac{1}{t_{k}}}\right)-\alpha_{2}^{1 / 2} \omega_{1}^{-\frac{1}{2}}(\log 2)^{-\frac{1}{2}} t_{k}^{-1}\left(\log \frac{1}{r}\right) \rightarrow 0
$$

for each $r \in\left(e^{-(\log 2) t_{k}^{2}}, 1\right)$. Especially, this convergence holds for $r \in\left(e^{-K t_{k}}, 1\right)$ with a positive constant $K$. Setting $r^{1 / t_{k}}=s$, and we have

$$
t_{k}^{\frac{1}{2}} v_{k}(s)-\alpha_{2}^{1 / 2} \omega_{1}^{-\frac{1}{2}}(\log 2)^{-\frac{1}{2}}\left(\log \frac{1}{s}\right) \rightarrow 0
$$

for each $s \in\left(e^{-K}, 1\right)$, in the sense of the pointwise convergence on $r=s^{t_{k}}$. If $t_{k}=o\left(c_{k}^{2}\right)$ holds, then

$$
o(1) c_{k} v_{k}(s)-\alpha_{2}^{1 / 2} \omega_{1}^{-\frac{1}{2}}(\log 2)^{-\frac{1}{2}}\left(\log \frac{1}{s}\right) \rightarrow 0 .
$$

This contradicts (13). Hence it follows that

$$
t_{k}=c_{k}^{2}(C+o(1))
$$

Combining (12), (14) and (15), we have

$$
t_{k}=c_{k}^{2}\left(\alpha_{2}^{-1} \omega_{1}(\log 2)^{-1}+o(1)\right), \quad \text { and } \quad w=\alpha_{2}^{1 / 2} \omega_{1}^{-1}\left(\log \frac{1}{|x|}\right)
$$

Consequently, we show that $w \neq 0$.

We note that

$$
\frac{\lambda_{k}}{c_{k}^{p}} \rightarrow 0
$$

Indeed, from Lemma 4.1 we have

$$
o(1)=\lambda_{k} \int v_{k}^{p} d x=\frac{\lambda_{k}}{c_{k}^{p}} \int_{B}\left(c_{k} v_{k}\right)^{p} d x=\frac{\lambda_{k}}{c_{k}^{p}}\left[\int_{B} w^{p} d x+o(1)\right] .
$$

We set $\delta_{k} \in(0,1)$ as the point such that

$$
e^{v_{k}\left(\delta_{k}\right)^{2}}-\frac{p}{2 \alpha_{2}^{p / 2}} \lambda_{k} v_{k}\left(\delta_{k}\right)^{p-2}=0 .
$$


Since $v_{k}$ is decreasing function with respect to $r$, this point $\delta_{k}$ is unique and

$$
\begin{gathered}
e^{v_{k}(r)^{2}}-\frac{p}{2 \alpha_{2}^{p / 2}} \lambda_{k} v_{k}(r)^{p-2} \geq 0 \quad \text { for } r \in\left[0, \delta_{k}\right] \\
e^{v_{k}(r)^{2}}-\frac{p}{2 \alpha_{2}^{p / 2}} \lambda_{k} v_{k}(r)^{p-2}<0 \quad \text { for } r \in\left(\delta_{k}, 1\right) .
\end{gathered}
$$

We observe that

$$
\frac{\alpha_{2}}{M_{k}} \int_{B} v_{k}^{2} e^{v_{k}^{2}} d x=\frac{\alpha_{2}}{M_{k}} \int_{B_{\delta_{k}}} v_{k}^{2} e^{v_{k}^{2}} d x+\frac{\alpha_{2}}{M_{k}} \int_{B_{1} \backslash B_{\delta_{k}}} v_{k}^{2} e^{v_{k}^{2}} d x=I_{1}+I_{2} .
$$

First, we show that

$$
I_{2} \leq \frac{C}{c_{k}^{4}}
$$

for some positive constant $C$. For $\theta>1$, we have

$$
e^{\left(\frac{c_{k}}{\theta}\right)^{2}}-\frac{p}{2 \alpha_{2}^{p / 2}} \lambda_{k}\left(\frac{c_{k}}{\theta}\right)^{p-2}>e^{\left(\frac{c_{k}}{\theta}\right)^{2}}-\frac{p}{2 \alpha_{2}^{p / 2} \theta^{p-2}} c_{k}^{2(p-1)} \rightarrow \infty
$$

as $k \rightarrow \infty$, where we used (16). Thus we have $v_{k}\left(\delta_{k}\right) \leq c_{k} / \theta$. We define $v_{k, \theta}$ in the same way as (10). Then by using Lemma 4.4, we have

$$
I_{2} \leq \frac{\alpha_{2}}{M_{k}} \int_{B} v_{k, \theta}^{2} e^{v_{k, \theta}^{2}} d x \leq \frac{\alpha_{2}}{M_{k}}\left(\int_{B} v_{k, \theta}^{\frac{2 \theta}{1-\theta}}\right)^{\frac{1-\theta}{\theta}}\left(\int_{B} e^{\theta v_{k, \theta}^{2}} d x\right)^{\frac{1}{\theta}} \leq \frac{C}{c_{k}^{4}} .
$$

Next, we show that

$$
I_{1} \leq 4 \pi+\frac{6 \pi}{c_{k}^{4}}+o\left(c_{k}^{-4}\right) .
$$

In order to prove this we apply the strategy of blow up analysis in [12].

We go back to the equation (4). Recall that as follows:

The function $\phi_{k}$ is defined by $\phi_{k}(y):=c_{k}\left(v_{k}\left(r_{k} y\right)-c_{k}\right)$ and $\phi_{k}$ satisfies

$$
-\Delta_{y} \phi_{k}=4\left(1+\frac{\phi_{k}}{c_{k}^{2}}\right) e^{\phi_{k}\left(2+\frac{\phi_{k}}{c_{k}^{2}}\right)}-\frac{2 p}{\alpha_{2}^{p / 2}} c_{k}^{p-2} e^{-c_{k}^{2}} \lambda_{k}\left(1+\frac{\phi_{k}}{c_{k}^{2}}\right)^{p-1} \text { in } B_{1 / r_{k}} .
$$

Then $\phi_{k} \rightarrow \phi_{\infty}:=-\log \left(1+|x|^{2}\right)$ in $C_{l o c}^{2}\left(\mathbb{R}^{2}\right)$ and $\phi_{\infty}$ satisfies

$$
-\Delta \phi_{\infty}=4 e^{2 \phi_{\infty}} \quad \text { in } \mathbb{R}^{2} \text {. }
$$

From the following lemma to Proposition 4.11, since the proofs are same as those in [13] and [12], we introduce only the statements. 
Lemma 4.8. Set $\eta_{k}:=c_{k}^{2}\left(\phi_{k}-\phi_{\infty}\right)$. Then there exists $\eta_{\infty}$ such that $\eta_{k} \rightarrow \eta_{\infty}$ in $C_{l o c}^{2}\left(\mathbb{R}^{N}\right), \eta_{\infty}$ is written by

$$
\eta_{\infty}(r)=\phi_{\infty}(r)+\frac{2 r^{2}}{1+r^{2}}-\frac{1}{2} \phi_{\infty}^{2}(r)+\frac{1-r^{2}}{1+r^{2}} \int_{1}^{1+r^{2}} \frac{\log t}{1-t} d t
$$

and $\eta_{\infty}$ is the unique solution of

$$
\left\{\begin{array}{l}
-\Delta w=4 e^{2 \phi_{\infty}}\left(\phi_{\infty}+\phi_{\infty}^{2}+2 w\right) \quad \text { in } \mathbb{R}^{2} \\
w(0)=w^{\prime}(0)=0
\end{array}\right.
$$

Moreover, $\eta_{\infty}=\phi_{\infty}+O(1)$ as $r \rightarrow \infty$ and $\int_{\mathbb{R}^{2}}-\Delta \eta_{\infty} d x=4 \pi$.

We write

$$
\phi_{k}=\phi_{\infty}+\frac{\eta_{\infty}}{c_{k}^{2}}+\frac{z_{k}}{c_{k}^{4}}
$$

On the second term in the right hand side, we have

$$
\frac{2 p}{\alpha_{2}^{p / 2}} c_{k}^{p-2} e^{-c_{k}^{2}} \lambda_{k}=o\left(c_{k}^{2 p-2} e^{-c_{k}^{2}}\right) .
$$

Thus we have

$$
\begin{aligned}
-\Delta \phi_{k}= & 4\left(1+\frac{\phi_{k}}{c_{k}^{2}}\right) e^{\phi_{k}\left(2+\frac{\phi_{k}}{c_{k}^{2}}\right)}+o\left(c_{k}^{2 p-2} e^{-c_{k}^{2}}\right) \\
= & 4 e^{2 \phi_{\infty}}\left[1+\frac{\phi_{\infty}+\phi_{\infty}^{2}+2 \eta_{\infty}}{c_{k}^{2}}+\frac{\eta_{\infty}+2 \eta_{\infty}^{2}+4 \phi_{\infty} \eta_{\infty}+2 \eta_{\infty} \phi_{\infty}^{2}+\phi_{\infty}^{3}+\frac{1}{2} \phi_{\infty}^{4}+2 z_{k}}{c_{k}^{4}}\right] \\
& +O\left(c_{k}^{-6}\right) .
\end{aligned}
$$

From this, we have $z_{k} \rightarrow z_{\infty}$ in $C_{l o c}^{2}\left(\mathbb{R}^{2}\right)$ and

$$
\left\{\begin{array}{l}
-\Delta z_{\infty}=4 e^{2 \phi_{\infty}}\left(\eta_{\infty}+2 \eta_{\infty}^{2}+4 \phi_{\infty} \eta_{\infty}+2 \eta_{\infty} \phi_{\infty}^{2}+\phi_{\infty}^{3}+\frac{1}{2} \phi_{\infty}^{4}+2 z_{k}\right) \quad \text { in } \mathbb{R}^{2} \\
z_{\infty}(0)=z_{\infty}^{\prime}(0)=0 .
\end{array}\right.
$$

We can observe that

$$
z_{\infty}(r)=\beta \log (r)+O(1) \quad \text { as } r \rightarrow \infty,
$$

where $\beta$ is represented by

$$
\beta=\frac{1}{2 \pi} \int_{\mathbb{R}^{2}}-\Delta z_{\infty} d x=6+\frac{\pi^{2}}{3} .
$$

We rewrite

$$
\phi_{k}=\phi_{\infty}+\frac{\eta_{\infty}}{c_{k}^{2}}+\frac{z_{\infty}}{c_{k}^{4}}+\frac{\tau_{k}}{c_{k}^{6}}
$$


Lemma 4.9. Let $0 \leq S \leq s_{k} \leq e^{c_{k}}$ and $\tau:\left[S, s_{k} \rightarrow \mathbb{R}\right]$ be given so that $\tau=o\left(c_{k}^{6}\right)$ uniformly on $\left[S, s_{k}\right]$. Set

$$
\phi=\phi_{\infty}+\frac{\eta_{\infty}}{c_{k}^{2}}+\frac{z_{\infty}}{c_{k}^{4}}+\frac{\tau}{c_{k}^{6}},
$$

and

$$
\mathcal{T}_{k}(r, \tau):=c_{k}^{6}\left[4\left(1+\frac{\phi}{c_{k}^{2}} e^{2 \phi+\frac{\phi}{c_{k}^{2}}}\right)+\Delta \phi_{\infty}+\frac{\Delta \eta_{\infty}}{c_{k}^{2}}+\frac{\Delta z_{\infty}}{c_{k}^{4}}\right] .
$$

Then

$$
\mathcal{T}_{k}(r, \tau)=4 e^{2 \phi_{\infty}}\left(2 \tau+o(1) \tau+O\left(c_{k}^{-2} \xi^{2}\right) \tau+O\left(\xi^{6}\right)\right)
$$

uniformly for $r \in\left[S, s_{k}\right]$, where

$$
\xi(r):=1+\log (1+r)
$$

Proposition 4.10. There exist $M>0, T>0$, and large constant $K(M, T)$ such that

$$
\left|\tau_{k}\right| \leq M \xi(r) \quad \text { for } r \in\left[0, e^{c_{k}}\right], \quad\left|\tau_{k}^{\prime}(r)\right| \leq \frac{M}{r} \quad \text { for } r \in\left[T, e^{c_{k}}\right],
$$

for any $k \geq K(M, T)$, where $\xi$ is defined in (21).

Proposition 4.11. Given a sequence $\left\{s_{k}\right\}$ with $s_{k} \in\left[c_{k}^{q}, e^{c_{k}}\right]$ for some $q>2$, we have

$$
\frac{\alpha_{2}}{M_{k}} \int_{B_{s_{k} r_{k}}} v_{k}^{2} e^{v_{k}^{2}} d x=4 \pi+\frac{4 \pi}{c_{k}^{4}}+o\left(c_{k}^{-4}\right) .
$$

Lemma 4.12. We have

$$
\phi_{k} \leq \phi_{\infty} \quad \text { in } \quad\left[\mu_{k}^{2}, \delta_{k} r_{k}^{-1}\right]
$$

for sufficient large $k$.

Proof. By Lemma 4.8 we have

$$
\eta_{\infty}\left(c_{k}^{2}\right) \leq-1, \quad \text { and } \quad \int_{\mathbb{R}^{2}} \Delta \eta_{\infty} d x<0
$$

Moreover, by the definition of $\mathcal{T}_{k}$ in Lemma 4.9 we can see that

$$
-\Delta \tau_{k}(r)=\mathcal{T}_{k}\left(r, \tau_{k}\right)
$$


and thus we have

$$
\sup _{\left(0, c_{k}^{2}\right)}\left|\frac{z_{\infty}}{c_{k}^{4}}+\frac{\tau_{k}}{c_{k}^{6}}\right|=o\left(\mu_{k}^{-2}\right), \quad \text { and } \quad \int_{B_{c_{k}^{2}}}\left|\Delta\left(\frac{z_{\infty}}{c_{k}^{4}}+\frac{\tau_{k}}{c_{k}^{6}}\right)\right| d x=o\left(c_{k}^{-2}\right)
$$

by Proposition 4.10. From these facts, it follows that

$$
\begin{aligned}
\int_{B_{c_{k}^{2}}} \Delta \phi_{k} d x & =\int_{B_{c_{k}^{2}}} \Delta \phi_{\infty} d x+\frac{1}{c_{k}^{2}} \int_{B_{c_{k}^{2}}} \Delta \eta_{\infty} d x+o\left(c_{k}^{-2}\right) \\
& =-4 \pi-\frac{c}{c_{k}^{2}}+o\left(c_{k}^{-2}\right) \\
& <-4 \pi .
\end{aligned}
$$

Since $\Delta \phi_{k} \leq 0$ in $\left[0, \delta_{k} r_{k}^{-1}\right]$, for $r \in\left[c^{2}, \delta_{k} r_{k}^{-1}\right]$ it follows that

$$
\int_{B_{r}} \Delta \phi_{k} d x \leq \int_{B_{c_{k}^{2}}} \Delta \phi_{k} d x<-4 \pi<\int_{B_{r}} \Delta \phi_{\infty} d x .
$$

By this inequality and $\phi_{k}\left(c_{k}^{2}\right)<\phi_{\infty}\left(c_{k}^{2}\right)$, we have $\phi_{k} \leq \phi_{\infty}$.

We can calculate in the same way as the proof of Proposition 14 in [12]. Therefore for $s_{k}$ given in Proposition 4.11 we have

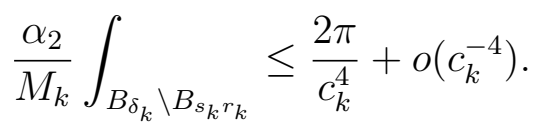

Hence combining Proposition 4.11 and (23), we finish the estimate of (19).

Finally, by (11), Proposition 4.7, (17), (18), and (19) we prove the Proposition 3.1.

\section{References}

[1] S. Adachi, K. Tanaka, Trudinger type inequalities in $\mathbb{R}^{N}$ and their best exponents. (English summary) Proc. Amer. Math. Soc. 128 (2000), no. 7, 2051-2057.

[2] Adimurthi, C. Tintarev, On compactness in the Trudinger-Moser inequality. (English summary) Ann. Sc. Norm. Super. Pisa Cl. Sci. (5) 13 (2014), no. 2, 399-416. 
[3] L. Carleson, S. A. Chang, On the existence of an extremal function for an inequality of J. Moser. (French summary) Bull. Sci. Math. (2) 110 (1986), no. 2, 113-127.

[4] D. Cassani, F. Sani, C. Tarsi, Equivalent Moser type inequalities in $\mathbb{R}^{2}$ and the zero mass case. (English summary) J. Funct. Anal. 267 (2014), no. 11, 4236-4263.

[5] D. G. de Figueiredo, J. M. do Ó, B. Ruf, On an inequality by N. Trudinger and J. Moser and related elliptic equations. (English summary) Comm. Pure Appl. Math. 55 (2002), no. 2, 135-152.

[6] M. Flucher, Extremal functions for the Trudinger-Moser inequality in 2 dimensions. Comment. Math. Helv. 67 (1992), no. 3, 471-497.

[7] M. Ishiwata, Existence and nonexistence of maximizers for variational problems associated with Trudinger-Moser type inequalities in $\mathbb{R}^{N}$. (English summary) Math. Ann. 351 (2011), no. 4, 781-804.

[8] Y. Li, B. Ruf, A sharp Trudinger-Moser type inequality for unbounded domains in $\mathbb{R}^{n}$. (English summary) Indiana Univ. Math. J. 57 (2008), no. $1,451-480$.

[9] K. C. Lin, Extremal functions for Moser's inequality. (English summary) Trans. Amer. Math. Soc. 348 (1996), no. 7, 2663-2671.

[10] P.-L. Lions, The concentration-compactness principle in the calculus of variations. The limit case. I. Rev. Mat. Iberoamericana 1 (1985), no. 1, 145-201.

[11] J. Moser, A sharp form of an inequality by N. Trudinger. Indiana Univ. Math. J. 20 (1970/71), 1077-1092.

[12] G. Mancini, L. Martinazzi, The Moser-Trudinger inequality and its extremals on a disk via energy estimates. (English summary) Calc. Var. Partial Differential Equations 56 (2017), no. 4, Art. 94, 26 pp.

[13] A. Malchiodi, L. Martinazzi, Critical points of the Moser-Trudinger functional on a disk. (English summary) J. Eur. Math. Soc. (JEMS) 16 (2014), no. 5, 893-908. 
[14] B. Ruf, A sharp Trudinger-Moser type inequality for unbounded domains in $\mathbb{R}^{2}$. (English summary) J. Funct. Anal. 219 (2005), no. 2, 340367.

[15] Y. Yang, Extremal functions for Moser-Trudinger inequalities on 2dimensional compact Riemannian manifolds with boundary. (English summary) Internat. J. Math. 17 (2006), no. 3, 313-330. 